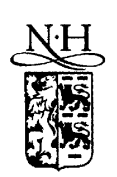

\title{
A better asymptotic profile of Rosenau-Burgers equation
}

\author{
Liping Liu *, Ming Mei
}

Department of Mathematical Sciences, University of Alberta, Edmonton, Alberta, Canada T6G 2 G1

\begin{abstract}
This paper studies the large-time behavior of the global solutions to the Cauchy problem for the Rosenau-Burgers (R-B) equation $u_{t}+u_{x x x x t}-\alpha u_{x x}+\left(u^{p+1} /(p+1)\right)_{x}=$ 0 . By the variable scaling method, we discover that the solution of the nonlinear parabolic equation $u_{t}-\alpha u_{x x}+\left(u^{p+1} /(p+1)\right)_{x}=0$ is a better asymptotic profile of the $\mathrm{R}-\mathrm{B}$ equation. The convergence rates of the $\mathrm{R}-\mathrm{B}$ equation to the asymptotic profile have been developed by the Fourier transform method with energy estimates. This result is better than the previous work [1,2] with zero as the asymptotic behavior. Furthermore, the numerical simulations on several test examples are discussed, and the numerical results confirm our theoretical results. (c) 2002 Elsevier Science Inc. All rights reserved.
\end{abstract}

Keywords: Rosenau-Burgers equation; Asymptotic profile; Convergence rates

\section{Introduction}

In this paper, we investigate the asymptotic behavior of the solution for the Rosenau-Burgers equation ( $\mathrm{R}-\mathrm{B})$ in the form

$$
\begin{cases}u_{t}+u_{x x x x t}-\alpha u_{x x}+\left(\frac{u^{p+1}}{p+1}\right)_{x}=0, & x \in \mathbf{R}, \quad t>0, \\ \left.u\right|_{t=0}=u_{0}(x) \rightarrow 0 & \text { as } x \rightarrow \pm \infty,\end{cases}
$$

where $\alpha$ is any given constant, $p \geqslant 1$ is integer.

\footnotetext{
${ }^{*}$ Corresponding author.

E-mail address: 1liu@math.ualberta.ca (L. Liu).
} 
Eq. (1.1) with $\alpha=0$ is called as the Rosenau equation proposed by Rosenau [3] for treating the dynamics of dense discrete systems in order to overcome the shortenings by the $\mathrm{KdV}$ equation, since the $\mathrm{KdV}$ equation describes unidimensional propagation of waves, but wave-wave and wave-wall interactions cannot be treated by it. Such a model were studied by Park [4] and by Chung and $\mathrm{Ha}$ [5] for the global existence of the solution to the IBVP. Eq. (1.1) with $\alpha>0$ is called the $\mathrm{R}-\mathrm{B}$ equation and somehow corresponds to the $\mathrm{KdV}-\mathrm{B}$ equation and the BBM-B equation, but it is given neither by Rosenau nor by Burgers. The Rosenau equation with the dissipative term $-\alpha u_{x x}$, or say, the $\mathrm{R}-$ $\mathrm{B}$ equation arises in some natural phenomena as for example, in bore propagation and in water waves.

The asymptotic behavior of the solution for the Cauchy problem (1.1) is studied in [1,2]. Where, it is proved that the solution $u(x, t)$ converges to 0 as fast as follows:

$$
\begin{gathered}
\|u(t)\|_{L^{2}} \leqslant C(1+t)^{-1 / 4}, \quad\|u(t)\|_{L^{\infty}} \leqslant C(1+t)^{-1 / 2} \\
\text { for all } t \geqslant 0 \quad \text { if } \int_{-\infty}^{\infty} u_{0}(x) \mathrm{d} x \neq 0
\end{gathered}
$$

and

$$
\begin{aligned}
& \|u(t)\|_{L^{2}} \leqslant C(1+t)^{-3 / 4}, \quad\|u(t)\|_{L^{\infty}} \leqslant C(1+t)^{-1} \\
& \text { for all } t \geqslant 0 \quad \text { if } \int_{-\infty}^{\infty} u_{0}(x) \mathrm{d} x=0 .
\end{aligned}
$$

In all the previous works, 0 is considered as the asymptotic state of the solution $u(x, t)$ for the $\mathrm{R}-\mathrm{B}$ equation. In this study, inspired by the works [6,7] on the BBM-B equations, we obtain a better asymptotic profile, i.e. a solution of its corresponding nonlinear parabolic equation, for the R-B equation. Furthermore, it is proved that the convergence to the asymptotic profile is faster than that to the zero.

\subsection{Notations}

For simplicity we introduce some notations. $C$ always denotes some positive constants without confusion. $\partial_{x}^{k} f:=\partial^{k} f / \partial x^{k} . L^{p}$ presents the Lebesgue integral space with the norm $\|\cdot\|_{L^{p}}$. Especially, $L^{2}$ is the square integral space with the norm $\|\cdot\|_{L^{2}}$, and $L^{\infty}$ is the essential bounded space with the norm $\|\cdot\|_{L^{\infty}} . H^{k}$ and $W^{k, p}$ denote the usual Sobolev spaces with the norms $\|\cdot\|_{H^{k}}$ and $\|\cdot\|_{W^{k, p}}$, respectively. Suppose that $f \in L^{1}(\mathbf{R}) \cap L^{2}(\mathbf{R})$, we define the Fourier transforms of $f$ as follows:

$$
F[f](\xi) \equiv \hat{f}(\xi)=\int_{\mathbf{R}} f(x) \mathrm{e}^{-\mathrm{i} x \xi} \mathrm{d} x .
$$


Let $T$ and $B$ be a positive constant and a Banach space, respectively. $C^{k}(0, T ; B)(k \geqslant 0)$ denotes the space of $B$-valued $k$-times continuously differentiable functions on $[0, T]$, and $L^{2}(0, T ; B)$ denotes the space of $B$-valued $L^{2}$ functions on $[0, T]$. The corresponding spaces of $B$-valued functions on $[0, \infty)$ are defined similarly.

\section{Reformulation and the main theorem}

Setting the following scalings to the variables:

$$
t \rightarrow \frac{t}{\varepsilon^{2}}, \quad x \rightarrow \frac{x}{\varepsilon}, \quad u \rightarrow \varepsilon^{m} u
$$

for some positive constants $m$ and $\varepsilon$, where $\varepsilon \ll 1$, we then scale the R-B equation (1.1) to

$$
u_{t}+\varepsilon^{4} u_{x x x x t}-\alpha u_{x x}+\varepsilon^{m p-1} u^{p} u_{x}=0 .
$$

Balancing the leading order terms, we choose $m=1 / p$, then Eq. (2.1) is reduced to

$$
u_{t}+\varepsilon^{4} u_{x x x x t}-\alpha u_{x x}+u^{p} u_{x}=0
$$

For $\varepsilon \ll 1$, neglecting the small term $\varepsilon^{4} u_{x x x x t}$ leads to the asymptotic state equation of the $\mathrm{R}-\mathrm{B}$ equation (1.1) as follows:

$$
u_{t}-\alpha u_{x x}+u^{p} u_{x}=0 .
$$

The solution of this parabolic solution should be a better asymptotic profile of Eq. (1.1).

By other choice of $m$ we derive other asymptotic state considered previously. Let $m$ be chosen as $m p<1$, we then rewrite Eq. (2.1) as

$$
\varepsilon^{1-m p}\left(u_{t}+\varepsilon^{4} u_{x x x x t}-\alpha u_{x x}\right)+u^{p} u_{x}=0 .
$$

For $\varepsilon \ll 1$, the term $\varepsilon^{1-m p}\left(u_{t}+\varepsilon^{4} u_{x x x x t}-\alpha u_{x x}\right)$ may be neglected so that the asymptotic equation of (2.2) is

$$
u^{p} u_{x}=0 \quad \text { i.e. }\left(\frac{u^{p+1}}{p+1}\right)_{x}=0 .
$$

Integrating it over $( \pm \infty, x]$ and noting that, formally $u( \pm \infty, t)=0$, we obtain $u^{p+1}(x, t)=u^{p+1}( \pm \infty, t)=0$, namely, $u(x, t)=0$. It means that the zero is the asymptotic state of the solution $u(x, t)$ of the $\mathrm{R}-\mathrm{B}$ equation. This case has been studied in [1,2].

Before our main theorem, we state the result for the solution of the nonlinear parabolic equation 


$$
\begin{aligned}
& \theta_{t}-\alpha \theta_{x x}+\theta^{p} \theta_{x}=0 \\
& \left.\theta\right|_{t=0}=\theta_{0}(x) \rightarrow 0 \quad \text { as } x \rightarrow \pm \infty .
\end{aligned}
$$

Theorem 2.1 [8-10]. Suppose that $\theta_{0}(x) \in H^{2}(\mathbf{R}) \cap L^{1}(\mathbf{R})$ holds. Then there exists a positive constant $\delta_{0}$ such that when $\left\|\theta_{0}\right\|_{L^{1}}+\left\|\theta_{0}\right\|_{H^{2}} \leqslant \delta_{0}$, then the Cauchy problem (2.3) has a unique global solution $\theta(x, t)$ with

$$
\theta(x, t) \in C\left(0, \infty ; H^{2}(\mathbf{R}) \cap L^{1}(\mathbf{R})\right) \cap L^{2}\left(0, \infty ; H^{1}(\mathbf{R})\right)
$$

and

$$
\begin{aligned}
\left\|\partial_{x}^{j} \theta(t)\right\|_{L^{q}}= & \mathcal{O}(1)\left(\left\|\theta_{0}\right\|_{L^{1}}+\left\|\theta_{0}\right\|_{H^{2}}\right) \\
& \times(1+t)^{-((j+1) q-1) /(2 q)}, \quad 1 \leqslant q \leqslant \infty .
\end{aligned}
$$

Furthermore, if $\theta_{0} \in L^{1}(\mathbf{R}) \cap H^{6}(\mathbf{R})$, then

$$
\left\|\partial_{x}^{j} \theta_{t}(t)\right\|_{L^{1}}=\mathcal{O}(1)\left(\left\|\theta_{0}\right\|_{L^{1}}+\left\|\theta_{0}\right\|_{H^{6}}\right)(1+t)^{-1-j / 2}, \quad j=0,1,2,3,4 .
$$

We now state our main result as follows.

Theorem 2.2. Suppose that $v_{0}(x)=\int_{-\infty}^{x}\left[u_{0}(y)-\theta_{0}(y)\right] \mathrm{d} y \in W^{3,1}(\mathbf{R})$ and $\theta_{0}(x) \in$ $L^{1}(\mathbf{R}) \cap H^{6}(\mathbf{R})$ hold. Let $\eta=\left\|\theta_{0}\right\|_{L^{1}}+\left\|\theta_{0}\right\|_{H^{6}}$. Then there exists a positive constant $\delta_{0}$ such that when $\left\|v_{0}\right\|_{W^{3,1}}+\eta \leqslant \delta_{0}$, then the Cauchy problem (1.1) has a unique global solution $u(x, t)$ with $u(x, t)-\theta(x, t) \in C\left(0, \infty ; H^{1}(\mathbf{R})\right)$ and satisfies that

(i) If $p=1$, for any $\sigma>0$, the following estimates hold:

$$
\begin{aligned}
& \|(u-\theta)(t)\|_{L^{2}} \leqslant C(1+t)^{-3 / 4+\sigma}, \\
& \left\|(u-\theta)_{x}(t)\right\|_{L^{2}} \leqslant C(1+t)^{-1+\sigma}, \\
& \|(u-\theta)(t)\|_{L^{\infty}} \leqslant C(1+t)^{-7 / 8+\sigma} .
\end{aligned}
$$

(ii) If $p \geqslant 2$, the convergence rates are much faster as follows:

$$
\begin{aligned}
& \|(u-\theta)(t)\|_{L^{2}} \leqslant C(1+t)^{-3 / 4}, \\
& \left\|(u-\theta)_{x}(t)\right\|_{L^{2}} \leqslant C(1+t)^{-5 / 4}, \\
& \|(u-\theta)(t)\|_{L^{\infty}} \leqslant C(1+t)^{-1} .
\end{aligned}
$$

Using $L^{2}, L^{\infty}$-results in the above theorem and the interposing inequality

$$
\|f\|_{L^{q}} \leqslant\|f\|_{L^{\infty}}^{(q-2) / q}\|f\|_{L^{2}}^{2 / q}, \quad 2 \leqslant q \leqslant \infty,
$$

immediately we obtain $L^{q}$-decay rates as follows: 
Corollary 2.3. Under the assumptions in Theorem 2.2 , for $2 \leqslant q \leqslant \infty$, it follows:

$$
\|(u-\theta)(t)\|_{L^{q}} \leqslant \begin{cases}C(1+t)^{-7 / 8+1 /(4 q)+\sigma}, & p=1 \\ C(1+t)^{-1+1 /(2 q),} & p \geqslant 2 .\end{cases}
$$

\section{Remark 2.4.}

1. In the case $\int_{-\infty}^{\infty} u_{0}(x) \mathrm{d} x \neq 0$ but $\int_{-\infty}^{\infty}\left(u_{0}(x)-\theta_{0}(x)\right) \mathrm{d} x=0$, our decay rates to the diffusion wave $\theta(x, t)$ are much better than that to the zero in [1,2]. This means that the solution $\theta(x, t)$ of the nonlinear parabolic Eq. (2.3) is a better asymptotic profile of the $\mathrm{R}-\mathrm{B}$ solution $u(x, t)$ than the zero.

2. In case $p=1$, it seems that the rate is not sufficient, and the optimal rate could be $(1+t)^{-1}$ as in the case $p \geqslant 2$. Our numerical simulation in Section 4 confirms it too. We expect more contributions on this problem.

\section{The proof of the main theorem}

From Eqs. (1.1) and (2.3), we have

$$
(u-\theta)_{t}-u_{x x x x t}-\alpha(u-\theta)_{x x}+\left(\frac{u^{p+1}}{p+1}-\frac{\theta^{p+1}}{p+1}\right)_{x}=0 .
$$

Since $\theta( \pm \infty, t)=0$, and we expect $u( \pm \infty, t)=0, u_{x}( \pm \infty, t)=0$, then after integrating (3.1) over $(-\infty, \infty)$, we have formally

$$
\frac{\mathrm{d}}{\mathrm{d} t} \int_{-\infty}^{\infty}[u(x, t)-\theta(x, t)] \mathrm{d} x=0 .
$$

Due to the essential assumption, we integrate (3.2) over $[0, t]$ with respect to $t$ to have

$$
\int_{-\infty}^{\infty}[u(x, t)-\theta(x, t)] \mathrm{d} x=\int_{-\infty}^{\infty}\left[u_{0}(x)-\theta_{0}(x)\right] \mathrm{d} x=0 .
$$

Therefore, it is natural to introduce

$$
v(x, t)=\int_{-\infty}^{x}[u(y, t)-\theta(y, t)] \mathrm{d} y, \quad \text { i.e. } v_{x}(x, t)=u(x, t)-\theta(x, t),
$$

which satisfies

$$
v_{x t}+v_{x x x x x t}-\theta_{x x x x t}-\alpha v_{x x x}+\left(\frac{\left(\theta+v_{x}\right)^{p+1}}{p+1}-\frac{\theta^{p+1}}{p+1}\right)_{x}=0 .
$$

Integrating the above equation over $(-\infty, x]$ with respect to $x$, and noting $u( \pm \infty, t)=0$, we obtain 


$$
\begin{aligned}
& v_{t}+v_{x x x x t}-\alpha v_{x x}-\theta_{x x x t}+\frac{\left(\theta+v_{x}\right)^{p+1}}{p+1}-\frac{\theta^{p+1}}{p+1}=0, \\
& \left.v\right|_{t=0}=\int_{-\infty}^{x}\left[u_{0}(y)-\theta_{0}(y)\right] \mathrm{d} y=v_{0}(x) .
\end{aligned}
$$

That is,

$$
\begin{aligned}
& v_{t}+v_{x x x x t}-\alpha v_{x x}=F_{p}(v), \\
& \left.v\right|_{t=0}=v_{0}(x),
\end{aligned}
$$

where

$$
\begin{aligned}
F_{p}(v) & =\theta_{x x x t}-\frac{1}{p+1}\left[\left(\theta+v_{x}\right)^{p+1}-\theta^{p+1}\right] \\
& =\theta_{x x x t}-\frac{1}{p+1} \sum_{j=0}^{p} a_{j} \theta^{j} v_{x}^{p+1-j}, \quad p \geqslant 1
\end{aligned}
$$

for some positive constants $a_{j}=\left(\begin{array}{c}j \\ p+1\end{array}\right)$. Thus, we get

$$
\begin{gathered}
\left|F_{p}(v)\right| \leqslant\left|\theta_{x x x t}\right|+\frac{1}{p+1} \sum_{j=0}^{p} a_{j}\left|\theta^{j} v_{x}^{p+1-j}\right|, \\
\left|\partial_{x} F_{p}(v)\right| \leqslant\left|\theta_{x x x x t}\right|+\left|v_{x}^{p} v_{x x}\right|+\frac{1}{p+1} \sum_{j=1}^{p} a_{j}\left\{j\left|\theta^{j-1} \theta_{x} v_{x}^{p+1-j}\right|\right. \\
\left.+(p+1-j)\left|\theta^{j} v_{x}^{p-j} v_{x x}\right|\right\} .
\end{gathered}
$$

Taking the Fourier transform to Eq. (3.5), we have

$$
\hat{v}_{t}+(\mathrm{i} \xi)^{4} \hat{v}_{t}-\alpha(\mathrm{i} \xi)^{2} \hat{v}=\widehat{F_{p}(v)}
$$

namely,

$$
\hat{v}_{t}+\frac{\alpha \xi^{2}}{1+\xi^{4}} \hat{v}=\frac{\widehat{F_{p}(v)}}{1+\xi^{4}},
$$

with the solution

$$
\hat{v}(\xi, t)=\mathrm{e}^{-B(\xi) t} \hat{v}_{0}(\xi)+\int_{0}^{t} \mathrm{e}^{-B(\xi)(t-s)} \frac{\widehat{F_{p}(v)}(\xi, s)}{1+\xi^{4}} \mathrm{~d} s,
$$

where

$$
B(\xi)=\frac{\alpha \xi^{2}}{1+\xi^{4}} .
$$

Then taking the inverse Fourier transform to the above resultant equation, we have 


$$
\begin{aligned}
v(x, t)= & \frac{1}{2 \pi} \int_{-\infty}^{\infty} \mathrm{e}^{\mathrm{i} \xi x} \mathrm{e}^{-B(\xi) t} \hat{v}_{0}(\xi) \mathrm{d} \xi \\
& +\frac{1}{2 \pi} \int_{0}^{t} \int_{-\infty}^{\infty} \mathrm{e}^{\mathrm{i} \xi x} \mathrm{e}^{-B(\xi)(t-s)} \frac{\widehat{F_{p}(v)}(\xi, s)}{1+\xi^{4}} \mathrm{~d} \xi \mathrm{d} s .
\end{aligned}
$$

Differentiating it with respect to $x$, we have

$$
\begin{aligned}
\partial_{x}^{j} v(x, t)= & \frac{1}{2 \pi} \int_{-\infty}^{\infty}(\mathrm{i} \xi)^{j} \mathrm{e}^{\mathrm{i} \xi x} \mathrm{e}^{-B(\xi) t} \hat{v}_{0}(\xi) \mathrm{d} \xi \\
& -\frac{1}{2 \pi} \int_{0}^{t} \int_{-\infty}^{\infty}(\mathrm{i} \xi)^{j} \mathrm{e}^{\mathrm{i} \xi x} \mathrm{e}^{-B(\xi)(t-s)} \frac{\widehat{F_{p}(v)}(\xi, s)}{1+\xi^{4}} \mathrm{~d} \xi \mathrm{d} s .
\end{aligned}
$$

We now define the solution spaces as follows, for any positive integer $p \geqslant 1$ and the given $\delta>0$,

$$
X_{p}^{\delta}=\left\{v \in C\left(0, \infty ; H^{2}(\mathbf{R})\right) \mid M_{p}(v) \leqslant \delta\right\},
$$

where

$$
\begin{aligned}
& M_{1}(v)=\sup _{0 \leqslant t \leqslant \infty}\left\{\sum_{j=0}^{1}(1+t)^{(2 j+1) / 4-\sigma}\left\|\partial_{x}^{j} v(t)\right\|_{L^{2}}+(1+t)^{1-\sigma}\left\|v_{x x}(t)\right\|_{L^{2}}\right\}, \\
& M_{p}(v)=\sup _{0 \leqslant t \leqslant \infty} \sum_{j=0}^{2}(1+t)^{(2 j+1) / 4}\left\|\partial_{x}^{j} v(t)\right\|_{L^{2}}, \quad p \geqslant 2 .
\end{aligned}
$$

Theorem 3.1. Under the same assumptions in Theorem 2.2, there exists a positive constant $\delta_{1}$, such that when $\left\|v_{0}\right\|_{W^{3,1}}+\eta<\delta_{1}$, then the Cauchy problem (3.5) has a unique global solution $v(x, t)$ satisfying $v(x, t) \in C\left(0, \infty ; H^{2}(\mathbf{R})\right)$. Furthermore, we have the following estimates:

1. When $p=1$, for any given $\sigma>0$, the solution $v(x, t)$ of (3.5) satisfies

$$
\begin{aligned}
& \sum_{i=0}^{1}(1+t)^{(2 j+1) / 4-\sigma}\left\|\partial_{x}^{j} v(t)\right\|_{L^{2}}+(1+t)^{1-\sigma}\left\|v_{x x}(t)\right\|_{L^{2}} \\
& \quad \leqslant C\left(\left\|v_{0}\right\|_{W^{3,1}}+\eta\right) .
\end{aligned}
$$

2. When $p \geqslant 2$, the solution $v(x, t)$ of (3.5) satisfies

$$
\sum_{j=0}^{2}(1+t)^{(2 j+1) / 4}\left\|\partial_{x}^{j} v(t)\right\|_{L^{2}} \leqslant C\left(\left\|v_{0}\right\|_{W^{3,1}}+\eta\right) \text {. }
$$

Once Theorem 3.1 is proved, by $u(x, t)-\theta(x, t)=v_{x}(x, t)$, it is easy to prove our main theorem, i.e. Theorem 2.2. Therefore, in the rest of this section, our main goal is to prove Theorem 3.1. Before we prove this theorem, we state several needed lemmas. 
Lemma 3.2 [1,2]. It holds

$$
\int_{-\infty}^{\infty} \frac{|\xi|^{j} \mathrm{e}^{-c B(\xi) t}}{\left(1+\xi^{4}\right)(1+|\xi|)^{j}} \mathrm{~d} \xi \leqslant C(1+t)^{-(j+1) / 2}
$$

for $j=0,1,2,3,4$, where $c$ is constant.

Lemma $3.3[11,12]$. Let $a>0$ and $b>0$ be constants. Then

$$
\begin{aligned}
& \int_{0}^{t}(1+t-s)^{-a}(1+s)^{-b} \mathrm{~d} s \\
& \quad \leqslant \begin{cases}C(1+t)^{-\min (a, b)} & \text { if } \max (a, b)>1, \\
C(1+t)^{-\min (a, b)} \log (2+t) & \text { if } \max (a, b)=1, \\
C(1+t)^{1-a-b} & \text { if } \max (a, b)<1 .\end{cases}
\end{aligned}
$$

Lemma $3.4[1,2]$. If $v_{0} \in W^{3,1}(\mathbf{R})$, then

$$
\left\|\frac{1}{2 \pi} \int_{-\infty}^{\infty}(\mathrm{i} \xi)^{j} \mathrm{e}^{\mathrm{i} \xi x} \mathrm{e}^{-B(\xi) t} \hat{v}_{0}(\xi) \mathrm{d} \xi\right\|_{L^{2}} \leqslant C\left\|v_{0}\right\|_{W^{j+1,1}}(1+t)^{-(2 j+1) / 4}
$$

for $j=0,1,2$.

The nonlinear term is estimated as follows.

Lemma 3.5. Suppose that $v(x, t) \in X_{p}^{\delta}$, then

(i) for $p=1$,

$$
\begin{aligned}
& \int_{0}^{t}\left\|\frac{1}{2 \pi} \int_{-\infty}^{\infty}(\mathrm{i} \xi)^{j} \mathrm{e}^{\mathrm{i} \xi x} \mathrm{e}^{-B(\xi)(t-s)} \frac{\widehat{F_{1}(v)}(\xi, s)}{1+\xi^{4}} \mathrm{~d} \xi\right\|_{L^{2}} \mathrm{~d} s \\
& \quad \leqslant C\left[\eta+(\eta+\delta)^{2}\right](1+t)^{-(2 j+1) / 4+\sigma}, \quad j=0,1 \\
& \int_{0}^{t}\left\|\frac{1}{2 \pi} \int_{-\infty}^{\infty}(\mathrm{i} \xi)^{2} \mathrm{e}^{\mathrm{i} \xi x} \mathrm{e}^{-B(\xi)(t-s)} \frac{\widehat{F_{1}(v)}(\xi, s)}{1+\xi^{4}} \mathrm{~d} \xi\right\|_{L^{2}} \mathrm{~d} s \\
& \quad \leqslant C\left[\eta+(\eta+\delta)^{2}\right](1+t)^{-1+\sigma} ;
\end{aligned}
$$

(ii) for $p \geqslant 2$,

$$
\begin{gathered}
\int_{0}^{t}\left\|\frac{1}{2 \pi} \int_{-\infty}^{\infty}(\mathrm{i} \xi)^{j} \mathrm{e}^{\mathrm{i} \xi x} \mathrm{e}^{-B(\xi)(t-s)} \frac{\widehat{F_{p}(v)}(\xi, s)}{1+\xi^{4}} \mathrm{~d} \xi\right\|_{L^{2}} \mathrm{~d} s \\
\quad \leqslant C\left[\eta+(\eta+\delta)^{p+1}\right](1+t)^{-(2 j+1) / 4}, \quad j=0,1,2 .
\end{gathered}
$$

Proof. Due to the definition of $X_{p}^{\delta}$ and Sobolev's inequality $\|f\|_{L^{\infty}} \leqslant \sqrt{2}\|f\|_{L^{2}}^{1 / 2} \cdot\left\|f_{x}\right\|_{L^{2}}^{1 / 2} \quad$ if $f \in H^{1}$, 
it is easy to obtain

(i) for $p=1$,

$$
\begin{aligned}
& \|v(t)\|_{L^{\infty}} \leqslant \sqrt{2}\|v(t)\|_{L^{2}}^{1 / 2}\left\|v_{x}(t)\right\|_{L^{2}}^{1 / 2} \leqslant \sqrt{2} \delta(1+t)^{-1 / 2+\sigma} \\
& \left\|v_{x}(t)\right\|_{L^{\infty}} \leqslant \sqrt{2}\left\|v_{x}(t)\right\|_{L^{2}}^{1 / 2}\left\|v_{x x}(t)\right\|_{L^{2}}^{1 / 2} \leqslant \sqrt{2} \delta(1+t)^{-7 / 8+\sigma}
\end{aligned}
$$

(ii) for $p \geqslant 2$,

$$
\begin{aligned}
& \|v(t)\|_{L^{\infty}} \leqslant \sqrt{2}\|v(t)\|_{L^{2}}^{1 / 2}\left\|v_{x}(t)\right\|_{L^{2}}^{1 / 2} \leqslant \sqrt{2} \delta(1+t)^{-1 / 2} \\
& \left\|v_{x}(t)\right\|_{L^{\infty}} \leqslant \sqrt{2}\left\|v_{x}(t)\right\|_{L^{2}}^{1 / 2}\left\|v_{x x}(t)\right\|_{L^{2}}^{1 / 2} \leqslant \sqrt{2} \delta(1+t)^{-1} .
\end{aligned}
$$

By inequality (3.6),

$$
\begin{aligned}
\sup _{\xi \in \mathbf{R}}\left|\widehat{F_{p}(v)}(\xi, s)\right| \leqslant & \int_{-\infty}^{\infty}\left|F_{p}(v)(x, s)\right| \mathrm{d} x \\
\leqslant & \int_{-\infty}^{\infty}\left\{\left|\theta_{x x x t}\right|+\frac{1}{p+1} \sum_{j=0}^{p} a_{j}\left|\theta^{j} v_{x}^{p+1-j}\right|\right\} \mathrm{d} x \\
\leqslant & \left\|\theta_{x x x t}(s)\right\|_{L^{1}}+\frac{1}{p+1} a_{p}\|\theta(s)\|_{L^{\infty}}^{p-1} \int_{-\infty}^{\infty}\left|\theta v_{x}\right| \mathrm{d} x \\
& +\frac{1}{p+1} \sum_{j=0}^{p-1} a_{j}\|\theta(s)\|_{L^{\infty}}^{j}\left\|v_{x}(s)\right\|_{L^{\infty}}^{p-1-j} \int_{-\infty}^{\infty}\left|v_{x}\right|^{2} \mathrm{~d} x \\
\leqslant & \left\|\theta_{x x x t}(s)\right\|_{L^{1}}+\frac{1}{p+1} a_{p}\|\theta(s)\|_{L^{\infty}}^{p-1}\|\theta(s)\|_{L^{2}}\left\|v_{x}(s)\right\|_{L^{2}} \\
& +\frac{1}{p+1} \sum_{j=0}^{p-1} a_{j}\|\theta(s)\|_{L^{\infty}}^{j}\left\|v_{x}(s)\right\|_{L^{\infty}}^{p-1-j}\left\|v_{x}(s)\right\|_{L^{2}}^{2} .
\end{aligned}
$$

Applying (3.17) and (3.18), the definition of $M_{p}(v)$ and Theorem 2.1 to the above inequality, we have

$$
\begin{aligned}
\sup _{\xi \in \mathbf{R}}\left|\widehat{F_{1}(v)}(\xi, s)\right| & \leqslant C\left\{\eta(1+s)^{-5 / 2}+\delta^{2}(1+s)^{-(3 / 2-2 \sigma)}+\eta \delta(1+s)^{-(1-\sigma)}\right\} \\
& \leqslant C\left\{\eta+(\eta+\delta)^{2}\right\}(1+s)^{-(1-\sigma)}
\end{aligned}
$$

by choosing $\sigma$ such that $0<\sigma \leqslant 1 / 2$, and

$$
\begin{aligned}
& \sup _{\xi \in \mathbf{R}}\left|\widehat{F_{p}(v)}(\xi, s)\right| \leqslant C\left\{(1+s)^{-5 / 2}+a_{p} \eta^{p} \delta(1+s)^{-(p+1) / 2}\right. \\
&\left.+\sum_{j=0}^{p-1} a_{j} \eta^{j} \delta^{p+1-j}(1+s)^{-(2 p-j+1) / 2}\right\} \\
& \leqslant C\left\{\eta(1+s)^{-3 / 2}+(\eta+\delta)^{p+1}(1+s)^{-(p+1) / 2}\right\} \\
& \leqslant C\left\{\eta+(\eta+\delta)^{p+1}\right\}(1+s)^{-3 / 2}, \quad p \geqslant 2,
\end{aligned}
$$


where for $p \geqslant 2$, we used $(p+1) / 2 \geqslant 3 / 2$ and $(2 p-j+1) / 2>(p+1) / 2$ for $0 \leqslant j \leqslant p-1$, which imply $(1+s)^{-(2 p-j+1) / 2}<(1+s)^{-(p+1) / 2} \leqslant(1+s)^{-3 / 2}$. Similarly, we estimate $\sup _{\xi \in \mathbf{R}}|\xi|\left|\widehat{F_{p}(v)}(\xi, s)\right|$ as follows.

$$
\begin{aligned}
\sup _{\xi \in \mathbf{R}}|\xi|\left|\widehat{F_{p}(v)}(\xi, s)\right| \leqslant & \int_{-\infty}^{\infty}\left|\partial_{x} F_{p}(v)(x, s)\right| \mathrm{d} x \\
\leqslant & \int_{-\infty}^{\infty}\left\{\left|\theta_{x x x x t}\right|+\left|v_{x x}\right| \cdot\left|v_{x}\right|^{p}+\frac{1}{p+1} \sum_{j=1}^{p} a_{j}\left[j\left|\theta^{j-1} \theta_{x} v_{x}^{p+1-j}\right|\right.\right. \\
& \left.\left.+(p+1-j)\left|\theta^{j} v_{x}^{p-j} v_{x x}\right|\right]\right\} \mathrm{d} x \\
\leqslant & \left\|\theta_{x x x x t}\right\|_{L^{1}}+\left\|v_{x}(s)\right\|_{L^{2}}^{p-1} \int_{-\infty}^{\infty}\left|v_{x} v_{x x}\right| \mathrm{d} x \\
& +\frac{1}{p+1} \sum_{j=1}^{p} a_{j}\left\{j\|\theta(s)\|_{L^{\infty}}^{j-1}\left\|v_{x}(s)\right\|_{L^{\infty}}^{p-j} \int_{-\infty}^{\infty}\left|\theta_{x} v_{x}\right| \mathrm{d} x\right. \\
& \left.+(p+1-j)\|\theta(s)\|_{L^{\infty}}^{j-1}\left\|v_{x}(s)\right\|_{L^{\infty}}^{p-j} \int_{-\infty}^{\infty}\left|\theta v_{x x}\right| \mathrm{d} x\right\} \\
\leqslant & \left\|\theta_{x x x x t}(s)\right\|_{L^{1}}+\left\|v_{x}(s)\right\|_{L^{2}}^{p-1}\left\|v_{x}(s)\right\|_{L^{2}}\left\|v_{x x}(s)\right\|_{L^{2}} \\
& +\frac{1}{p+1} \sum_{j=1}^{p} a_{j}\left\{j\|\theta(s)\|_{L^{\infty}}^{j-1}\left\|v_{x}(s)\right\|_{L^{\infty}}^{p-j}\left\|\theta_{x}(s)\right\|_{L^{2}}\left\|v_{x}(s)\right\|_{L^{2}}\right. \\
& \left.+(p+1-j)\|\theta(s)\|_{L^{\infty}}^{j-1}\left\|v_{x}(s)\right\|_{L^{\infty}}^{p-j}\|\theta(s)\|_{L^{2}}\left\|v_{x x}(s)\right\|_{L^{2}}\right\} .
\end{aligned}
$$

Depending on the value of $p$, we have

$$
\begin{aligned}
\sup _{\xi \in \mathbf{R}}|\xi|\left|\widehat{F_{1}(v)}(\xi, s)\right| & \leqslant C\left\{\eta(1+s)^{-3}+\delta^{2}(1+s)^{-2(1-\sigma)}+\eta \delta(1+s)^{-(3 / 2-\sigma)}\right\} \\
& \leqslant C\left\{\eta+(\eta+\delta)^{2}\right\}(1+s)^{-(3 / 2-\sigma)}, \quad 0<\sigma \leqslant 1 / 2,
\end{aligned}
$$

$$
\begin{aligned}
\sup _{\xi \in \mathbf{R}}\left|\xi \| \widehat{F_{p}(v)}(\xi, s)\right| \leqslant C & \left\{(1+s)^{-3}+\delta^{p+1}(1+s)^{-(p+1)}\right. \\
& +\frac{1}{p+1} \sum_{j=1}^{p} a_{j}\left[j \eta^{j} \delta^{p-j+1}(1+s)^{-(2 p-j+2) / 2}\right. \\
& \left.\left.+(p+1-j) \eta^{j} \delta^{p-j+1}(1+s)^{-(2 p-j+2) / 2}\right]\right\} \\
\leqslant C & \left\{\eta(1+s)^{-3}+\delta^{p+1}(1+s)^{-(p+1)}\right.
\end{aligned}
$$




$$
\begin{gathered}
\left.+\sum_{j=1}^{p} a_{j} \eta^{j} \delta^{p-j+1}(1+s)^{-(p+2) / 2}\right\} \\
\leqslant C\left\{\eta+(\eta+\delta)^{p+1}\right\}(1+s)^{-2}, \quad p \geqslant 2,
\end{gathered}
$$

where for $p \geqslant 2$ and $1 \leqslant j \leqslant p$, we used $p+1 \geqslant 3$ and $(2 p-j+2) / 2 \geqslant$ $(p+2) / 2 \geqslant 2$, which imply $(1+s)^{-(p+1)}<(1+s)^{-2}$ and $(1+s)^{-(2 p-j+2) / 2} \leqslant$ $(1+s)^{-(p+2) / 2} \leqslant(1+s)^{-2}$.

Making use of Parseval's equality, we have

$$
\begin{aligned}
\int_{0}^{t} & \left\|\frac{1}{2 \pi} \int_{-\infty}^{\infty} \mathrm{e}^{\mathrm{i} \xi x} \frac{\mathrm{e}^{-B(\xi)(t-s)}}{1+\xi^{4}} \widehat{F_{p}(v)}(\xi, s) \mathrm{d} \xi\right\|_{L^{2}} \mathrm{~d} s \\
= & \int_{0}^{t}\left\|\frac{\mathrm{e}^{-B(\xi)(t-s)}}{1+\xi^{4}} \widehat{F_{p}(v)}(\xi, s)\right\|_{L^{2}} \mathrm{~d} s \\
= & \int_{0}^{t}\left(\int_{-\infty}^{\infty} \frac{\mathrm{e}^{-2 B(\xi)(t-s)}}{\left(1+\xi^{4}\right)^{2}}\left|\widehat{F_{p}(v)}(\xi, s)\right|^{2} \mathrm{~d} \xi\right)^{1 / 2} \mathrm{~d} s \\
& \leqslant \int_{0}^{t} \sup _{\xi \in \mathbf{R}}\left|\widehat{F_{p}(v)}(\xi, s)\right|\left(\int_{-\infty}^{\infty} \frac{\mathrm{e}^{-2 B(\xi)(t-s)}}{\left(1+\xi^{4}\right)^{2}} \mathrm{~d} \xi\right)^{1 / 2} \mathrm{~d} s .
\end{aligned}
$$

Noting (3.20) and (3.21), Lemmas 3.2 and 3.3, we obtain

$$
\begin{aligned}
& \int_{0}^{t}\left\|\frac{1}{2 \pi} \int_{-\infty}^{\infty} \mathrm{e}^{\mathrm{i} \xi x} \frac{\mathrm{e}^{-B(\xi)(t-s)}}{1+\xi^{4}} \widehat{F_{1}(v)}(\xi, s) \mathrm{d} \xi\right\|_{L^{2}} \mathrm{~d} s \\
& \leqslant \leqslant\left\{\eta+(\eta+\delta)^{2}\right\} \int_{0}^{t}(1+s)^{-(1-\sigma)}(1+t-s)^{-1 / 4} \mathrm{~d} s \\
& \leqslant \leqslant\left\{\eta+(\eta+\delta)^{2}\right\}(1+t)^{1-(1-\sigma)-1 / 4} \\
& \leqslant \leqslant\left\{\eta+(\eta+\delta)^{2}\right\}(1+t)^{-(1 / 4-\sigma)}, \quad 0<\sigma \leqslant 1 / 2 \\
& \int_{0}^{t} \| \frac{1}{2 \pi} \int_{-\infty}^{\infty} \mathrm{e}^{\mathrm{i} \xi x} \frac{\mathrm{e}^{-B(\xi)(t-s)} \widehat{1+\xi^{4}} \widehat{F_{p}(v)}(\xi, s) \mathrm{d} \xi \|_{L^{2}} \mathrm{~d} s}{\leqslant C\left\{\eta+(\eta+\delta)^{p+1}\right\} \int_{0}^{t}(1+s)^{-3 / 2}(1+t-s)^{-1 / 4} \mathrm{~d} s} \\
& \leqslant C\left\{\eta+(\eta+\delta)^{p+1}\right\}(1+t)^{-1 / 4}, \quad p \geqslant 2 .
\end{aligned}
$$


Similarly, we have

$$
\begin{aligned}
\int_{0}^{t} & \left\|\frac{1}{2 \pi} \int_{-\infty}^{\infty} \mathrm{i} \xi \mathrm{e}^{\mathrm{i} \xi x} \frac{\mathrm{e}^{-B(\xi)(t-s)}}{1+\xi^{4}} \widehat{F_{p}(v)}(\xi, s) \mathrm{d} \xi\right\|_{L^{2}} \mathrm{~d} s \\
= & \int_{0}^{t}\left\|\mathrm{i} \xi \frac{\mathrm{e}^{-B(\xi)(t-s)}}{1+\xi^{4}} \widehat{F_{p}(v)}(\xi, s) \mid\right\|_{L^{2}} \mathrm{~d} s \\
= & \int_{0}^{t}\left(\int_{-\infty}^{\infty} \frac{|\xi|^{2} \mathrm{e}^{-2 B(\xi)(t-s)}}{\left(1+\xi^{4}\right)^{2}}\left|\widehat{F_{p}(v)}(\xi, s)\right|^{2} \mathrm{~d} \xi\right)^{1 / 2} \mathrm{~d} s \\
\leqslant & \int_{0}^{t} \sup _{\xi \in \mathbf{R}}\left|\widehat{F_{p}(v)}(\xi, s)\right|\left(\int_{-\infty}^{\infty} \frac{|\xi|^{2} \mathrm{e}^{-2 B(\xi)(t-s)}}{\left(1+\xi^{4}\right)^{2}} \mathrm{~d} \xi\right)^{1 / 2} \mathrm{~d} s .
\end{aligned}
$$

Using (3.20) and (3.21), Lemmas 3.2 and 3.3, we obtain

$$
\begin{aligned}
& \int_{0}^{t}\left\|\frac{1}{2 \pi} \int_{-\infty}^{\infty} \mathrm{i} \xi \mathrm{e}^{\mathrm{i} \xi x} \frac{\mathrm{e}^{-B(\xi)(t-s)}}{1+\xi^{4}} \widehat{F_{1}(v)}(\xi, s) \mathrm{d} \xi\right\|_{L^{2}} \mathrm{~d} s \\
& \leqslant\left.\leqslant C \eta+(\eta+\delta)^{2}\right\} \int_{0}^{t}(1+s)^{-(1-\sigma)}(1+t-s)^{-3 / 4} \mathrm{~d} s \\
& \leqslant C\left\{\eta+(\eta+\delta)^{2}\right\}(1+t)^{1-(1-\sigma)-3 / 4} \\
&=C\left\{\eta+(\eta+\delta)^{2}\right\}(1+t)^{-(3 / 4-\sigma)}, \quad 0<\sigma \leqslant 1 / 2 \\
& \int_{0}^{t}\left\|\frac{1}{2 \pi} \int_{-\infty}^{\infty} \mathrm{i} \xi \mathrm{e}^{\mathrm{i} \xi x} \frac{\mathrm{e}^{-B(\xi)(t-s)}}{1+\xi^{4}} \widehat{F_{p}(v)}(\xi, s) \mathrm{d} \xi\right\|_{L^{2}} \mathrm{~d} s \\
& \leqslant C\left\{\eta+(\eta+\delta)^{p+1}\right\} \int_{0}^{t}(1+s)^{-3 / 2}(1+t-s)^{-3 / 4} \mathrm{~d} s \\
& \leqslant C\left\{\eta+(\eta+\delta)^{p+1}\right\}(1+t)^{-3 / 4}, \quad p \geqslant 2 .
\end{aligned}
$$

Furthermore,

$$
\begin{aligned}
\int_{0}^{t} & \left\|\frac{1}{2 \pi} \int_{-\infty}^{\infty}(\mathrm{i} \xi)^{2} \mathrm{e}^{\mathrm{i} \xi x} \frac{\mathrm{e}^{-B(\xi)(t-s)}}{1+\xi^{4}} \widehat{F_{p}(v)}(\xi, s) \mathrm{d} \xi\right\|_{L^{2}} \mathrm{~d} s \\
= & \int_{0}^{t}\left\|(\mathrm{i} \xi)^{2} \frac{\mathrm{e}^{-B(\xi)(t-s)}}{1+\xi^{4}} \widehat{F_{p}(v)}(\xi, s)\right\|_{L^{2}} \mathrm{~d} s \\
= & \int_{0}^{t}\left(\int_{-\infty}^{\infty} \frac{|\xi|^{4} \mathrm{e}^{-2 B(\xi)(t-s)}}{\left(1+\xi^{4}\right)^{2}}\left|\widehat{F_{p}(v)}(\xi, s)\right|^{2} \mathrm{~d} \xi\right)^{1 / 2} \mathrm{~d} s \\
& \leqslant \int_{0}^{t} \sup _{\xi \in \mathbf{R}}\left((1+|\xi|)\left|\widehat{F_{p}(v)}(\xi, s)\right|\right)\left(\int_{-\infty}^{\infty} \frac{|\xi|^{4} \mathrm{e}^{-2 B(\xi)(t-s)}}{\left(1+\xi^{4}\right)(1+|\xi|)^{4}} \mathrm{~d} \xi\right)^{1 / 2} \mathrm{~d} s .
\end{aligned}
$$


Using (3.20), (3.21), (3.23) and (3.24), Lemmas 3.2 and 3.3, we have

$$
\begin{aligned}
& \int_{0}^{t} \| \frac{1}{2 \pi} \int_{-\infty}^{\infty}(\mathrm{i} \xi)^{2} \mathrm{e}^{\mathrm{i} \xi x} \frac{\mathrm{e}^{-B(\xi)(t-s)} \widehat{F_{1}(v)}(\xi, s) \mathrm{d} \xi \|_{L^{2}} \mathrm{~d} s}{1+\xi^{4}} \\
& \leqslant C\left\{\eta+(\eta+\delta)^{2}\right\} \int_{0}^{t}\left\{(1+s)^{-(1-\sigma)}+(1+s)^{-(3 / 2-\sigma)}\right\}(1+t-s)^{-5 / 4} \mathrm{~d} s \\
& \leqslant C\left\{\eta+(\eta+\delta)^{2}\right\} \int_{0}^{t}(1+s)^{-(1-\sigma)}(1+t-s)^{-5 / 4} \mathrm{~d} s \\
& \leqslant C\left\{\eta+(\eta+\delta)^{2}\right\}(1+t)^{-(1-\sigma)}, \quad 0<\sigma \leqslant 1 / 2 . \\
& \int_{0}^{t} \| \frac{1}{2 \pi} \int_{-\infty}^{\infty}(\mathrm{i} \xi)^{2} \mathrm{e}^{\mathrm{i} \xi x} \frac{\mathrm{e}^{-B(\xi)(t-s)} \widehat{1+\xi^{4}} \widehat{F_{p}(v)}(\xi, s) \mathrm{d} \xi \|_{L^{2}} \mathrm{~d} s}{\leqslant C\left\{\eta+(\eta+\delta)^{p+1}\right\} \int_{0}^{t}\left\{(1+s)^{-3 / 2}+(1+s)^{-2}\right\}(1+t-s)^{-5 / 4} \mathrm{~d} s} \\
& \leqslant C\left\{\eta+(\eta+\delta)^{p+1}\right\} \int_{0}^{t}(1+s)^{-3 / 2}(1+t-s)^{-5 / 4} \mathrm{~d} s \\
& \leqslant C\left\{\eta+(\eta+\delta)^{p+1}\right\}(1+t)^{-5 / 4}, \quad p \geqslant 2 .
\end{aligned}
$$

With (3.26), (3.27), (3.29), (3.30), (3.32) and (3.33), we finish the proof of the lemma.

Lemma 3.6. For $v_{1}(x, t), v_{2}(x, t) \in X_{p}^{\delta}$, the followings are valid:

$$
\begin{aligned}
& \sup _{\xi \in \mathbf{R}}\left|\widehat{F_{1}} \widehat{\left(v_{1}\right)}(\xi, s)-\widehat{F_{1}} \widehat{\left(v_{2}\right)}(\xi, s)\right| \leqslant C(\eta+\delta) M\left(v_{1}-v_{2}\right)(1+s)^{-1+\sigma}, \\
& \sup _{\xi \in \mathbf{R}}\left|\widehat{F_{p}} \widehat{\left(v_{1}\right)}(\xi, s)-\widehat{F_{p}} \widehat{\left(v_{2}\right)}(\xi, s)\right| \leqslant C(\eta+\delta)^{p} M\left(v_{1}-v_{2}\right)(1+s)^{-3 / 2} \\
& \quad \text { for } p \geqslant 2, \\
& \sup _{\xi \in \mathbf{R}}|\xi|\left|\widehat{F_{1}} \widehat{\left(v_{1}\right)}(\xi, s)-\widehat{F_{1}\left(v_{2}\right)}(\xi, s)\right| \leqslant C(\eta+\delta) M\left(v_{1}-v_{2}\right)(1+s)^{-5 / 4+\sigma}, \\
& \sup _{\xi \in \mathbf{R}}|\xi|\left|\widehat{F_{p}\left(v_{1}\right)}(\xi, s)-\widehat{F_{p}\left(\widehat{\left.v_{2}\right)}\right)}(\xi, s)\right| \leqslant C(\eta+\delta)^{p} M\left(v_{1}-v_{2}\right)(1+s)^{-2} \\
& \quad \text { for } p \geqslant 2 .
\end{aligned}
$$

Proof. For $p=1$, we have 


$$
\begin{aligned}
& \sup _{\xi \in \mathbf{R}}\left|\widehat{F_{1}} \widehat{\left(v_{1}\right)}(\xi, s)-\widehat{F_{1}} \widehat{\left(v_{2}\right)}(\xi, s)\right| \\
& \quad \leqslant \int_{-\infty}^{\infty}\left(\left|\theta\left(v_{1 x}-v_{2 x}\right)\right|+\frac{1}{2}\left|v_{1 x}+v_{2 x} \| v_{1 x}-v_{2 x}\right|\right) \mathrm{d} x \\
& \leqslant\|\theta(s)\|_{L^{2}}\left\|\left(v_{1 x}-v_{2 x}\right)(s)\right\|_{L^{2}}+\frac{1}{2}\left\|\left(v_{1 x}+v_{2 x}\right)(s)\right\|_{L^{2}}\left\|\left(v_{1 x}-v_{2 x}\right)(s)\right\|_{L^{2}} \\
& \leqslant\left(\|\theta(s)\|_{L^{2}}+\frac{1}{2}\left\|v_{1 x}(s)\right\|_{L^{2}}+\frac{1}{2}\left\|v_{2 x}(s)\right\|_{L^{2}}\right)\left\|\left(v_{1 x}-v_{2 x}\right)(s)\right\|_{L^{2}} \\
& \leqslant C\left(\eta(1+s)^{-1 / 4}+\delta(1+s)^{-3 / 4+\sigma}\right) M\left(v_{1}-v_{2}\right)(1+s)^{-3 / 4+\sigma} \\
& \leqslant C(\eta+\delta)(1+s)^{-1 / 4} M\left(v_{1}-v_{2}\right)(1+s)^{-3 / 4+\sigma} \\
& \leqslant C(\eta+\delta) M\left(v_{1}-v_{2}\right)(1+s)^{-1+\sigma}
\end{aligned}
$$

and

$$
\begin{aligned}
& \sup _{\xi \in \mathbf{R}}|\xi|\left|\widehat{F_{1}\left(v_{1}\right)}(\xi, s)-\widehat{F_{1}\left(v_{2}\right)}(\xi, s)\right| \\
& \leqslant \int_{-\infty}^{\infty}\left|F_{1 x}\left(v_{1}\right)(x, s)-F_{1 x}\left(v_{2}\right)(x, s)\right| \mathrm{d} x \\
& \leqslant \int_{-\infty}^{\infty}\left|\theta_{x}\left(v_{1 x}-v_{2 x}\right)\right|+\left|\theta\left(v_{1 x x}-v_{2 x x}\right)\right|+\left|v_{1 x} v_{1 x x}-v_{2 x} v_{2 x x}\right| \mathrm{d} x \\
& \leqslant\left\|\theta_{x}(s)\right\|_{L^{2}}\left\|\left(v_{1 x}-v_{2 x}\right)(s)\right\|_{L^{2}}+\|\theta(s)\|_{L^{2}}\left\|\left(v_{1 x x}-v_{2 x x}\right)(s)\right\|_{L^{2}} \\
&+\left\|v_{1 x}(s)\right\|_{L^{2}}\left\|\left(v_{1 x x}-v_{2 x x}\right)(s)\right\|_{L^{2}}+\left\|v_{2 x x}(s)\right\|_{L^{2}}\left\|\left(v_{1 x}-v_{2 x}\right)(s)\right\|_{L^{2}} \\
& \leqslant\left(\left\|\theta_{x}(s)\right\|_{L^{2}}+\left\|v_{2 x x}(s)\right\|_{L^{2}}\right)\left\|\left(v_{1 x}-v_{2 x}\right)(s)\right\|_{L^{2}} \\
&+\left(\|\theta(s)\|_{L^{2}}+\left\|v_{1 x}(s)\right\|_{L^{2}}\right)\left\|\left(v_{1 x x}-v_{2 x x}\right)(s)\right\|_{L^{2}} \\
& \leqslant C\left\{\left(\eta(1+s)^{-3 / 4}+\delta(1+s)^{-1+\sigma}\right) M\left(v_{1}-v_{2}\right)(1+s)^{-3 / 4+\sigma}\right. \\
&\left.+\left(\eta(1+s)^{-1 / 4}+\delta(1+s)^{-3 / 4+\sigma}\right) M\left(v_{1}-v_{2}\right)(1+s)^{-1+\sigma}\right\} \\
& \leqslant C(\eta+\delta) M\left(v_{1}-v_{2}\right)\left\{(1+s)^{-3 / 2+\sigma}+(1+s)^{-7 / 4+2 \sigma}\right. \\
&\left.+(1+s)^{-5 / 4+\sigma}+(1+s)^{-7 / 4+2 \sigma}\right\} \\
& \leqslant C(\eta+\delta) M\left(v_{1}-v_{2}\right)(1+s)^{-5 / 4+\sigma} .
\end{aligned}
$$

For $p \geqslant 2, \sup _{\xi \in \mathbf{R}}\left|\widehat{F_{p}\left(v_{1}\right)}(\xi, s)-\widehat{F_{p}\left(v_{2}\right)}(\xi, s)\right|$ can be estimated as follows: 


$$
\begin{aligned}
\sup _{\xi \in \mathbf{R}} \mid & \widehat{F_{p}\left(v_{1}\right)}(\xi, s)-\widehat{F_{p}\left(v_{2}\right)}(\xi, s) \mid \\
\leqslant & \int_{-\infty}^{\infty}\left|F_{p}\left(v_{1}\right)(x, s)-F_{p}\left(v_{2}\right)(x, s)\right| \mathrm{d} x \\
\leqslant & \int_{-\infty}^{\infty} \frac{1}{p+1} \sum_{j=0}^{p} a_{j}\left|\theta^{j} v_{1 x}^{p+1-j}-\theta^{j} v_{2 x}^{p+1-j}\right| \mathrm{d} x \\
\leqslant & \frac{1}{p+1} \int_{-\infty}^{\infty}\left|a_{p} \theta\right|^{p}\left|v_{1 x}-v_{2 x}\right| \mathrm{d} x+\frac{1}{p+1} \sum_{j=0}^{p-1} a_{j} \int_{-\infty}^{\infty}|\theta|^{j}\left|v_{1 x}^{p+1-j}-v_{2 x}^{p+1-j}\right| \mathrm{d} x \\
\leqslant & \frac{1}{p+1}\left\{a_{p}\|\theta(s)\|_{L^{\infty}}^{p-1}\|\theta(s)\|_{L^{2}}\left\|\left(v_{1 x}-v_{2 x}\right)(s)\right\|_{L^{2}}\right. \\
& \left.+\sum_{j=0}^{p-1} a_{j}\|\theta(s)\|_{L^{\infty}}^{j}\left\|\left(v_{1 x}-v_{2 x}\right)(s)\right\|_{L^{2}} \sum_{i=0}^{p-j}\left\|v_{1 x}(s)\right\|_{L^{2}}^{i}\left\|v_{2 x}(s)\right\|_{L^{2}}^{p-j-i}\right\} \\
\leqslant & \frac{C}{p+1}\left\{a_{p} \eta^{p} M\left(v_{1}-v_{2}\right)(1+s)^{-(p+1) / 2}\right. \\
& \left.+\sum_{j=0}^{p-1} a_{j} \eta^{j} \delta^{p-j} M\left(v_{1}-v_{2}\right)(1+s)^{-(3 p+3-j) / 4}(p-j)\right\} \\
\leqslant & \frac{C}{p+1}\left\{a_{p} \eta^{p}+\sum_{j=0}^{p-1} a_{j}(p-j) \eta^{j} \delta^{p-j}\right\} M\left(v_{1}-v_{2}\right)(1+s)^{-(p+1) / 2} \\
\leqslant & C(\eta+\delta)^{p} M\left(v_{1}-v_{2}\right)(1+s)^{-3 / 2},
\end{aligned}
$$

where we used $0 \leqslant j \leqslant p-1$ and $p \geqslant 2$ which imply $-(3 p+3-j) / 4 \leqslant-(3 p+$ $3-(p-1)) / 4 \leqslant-(p+1) / 2 \leqslant-3 / 2$. And

$$
\begin{aligned}
\sup _{\xi \in \mathbf{R}}|\xi|\left|F_{p} \widehat{\left(v_{1}\right)}(\xi, s)-\widehat{F_{p}\left(v_{2}\right)}(\xi, s)\right| \\
\leqslant \int_{-\infty}^{\infty}\left|\partial_{x} F_{p}\left(v_{1}\right)(x, s)-\partial_{x} F_{p}\left(v_{2}\right)(x, s)\right| \mathrm{d} x \\
\leqslant \int_{-\infty}^{\infty}\left\{\left|v_{1 x x} v_{1 x}^{p}-v_{2 x x} v_{2 x}^{p}\right|+\frac{1}{p+1} a_{p}\left[p\left|\theta^{p-1} \theta_{x}\left(v_{1 x}-v_{2 x}\right)\right|\right.\right. \\
\left.\quad+\left|\theta^{p}\left(v_{1 x x}-v_{2 x x}\right)\right|\right]+\frac{1}{p+1} \sum_{j=1}^{p-1} a_{j}\left[j\left|\theta^{j-1} \theta_{x}\left(v_{1 x}^{p+1-j}-v_{2 x}^{p+1-j}\right)\right|\right. \\
\left.\left.\quad+(p+1-j)\left|\theta^{j}\left(v_{1 x}^{p-j} v_{1 x x}-v_{2 x}^{p-j} v_{2 x x}\right)\right|\right]\right\} \mathrm{d} x
\end{aligned}
$$




$$
\begin{aligned}
\leqslant & \left\|\left(v_{1 x x} v_{1 x}^{p}-v_{2 x x} v_{2 x}^{p}\right)(s)\right\|_{L^{1}}+\frac{1}{p+1} a_{p}\left\{p\left\|\theta^{p-1}(s) \theta_{x}(s)\left(v_{1 x}-v_{2 x}\right)(s)\right\|_{L^{1}}\right. \\
& \left.+\left\|\theta(s)^{p}\left(v_{1 x x}-v_{2 x x}\right)(s)\right\|_{L^{1}}\right\} \\
& +\frac{1}{p+1} \sum_{j=1}^{p-1} a_{j}\left\{j\left\|\theta^{j-1}(s) \theta_{x}(s)\left(v_{1 x}^{p+1-j}-v_{2 x}^{p+1-j}\right)(s)\right\|_{L^{1}}\right. \\
& \left.+(p+1-j)\left\|\theta(s)^{j}\left(v_{1 x}^{p-j} v_{1 x x}-v_{2 x}^{p-j} v_{2 x x}\right)(s)\right\|_{L^{1}}\right\} .
\end{aligned}
$$

Since we have

$$
\begin{aligned}
\left\|\left(v_{1 x x} v_{1 x}^{p}-v_{2 x x} v_{2 x}^{p}\right)(s)\right\|_{L^{1}} & \\
\leqslant & \left\|\left(v_{1 x x} v_{1 x}^{p}-v_{1 x x} v_{2 x}^{p}\right)(s)\right\|_{L^{1}}+\left\|\left(v_{1 x x} v_{2 x}^{p}-v_{2 x x} v_{2 x}^{p}\right)(s)\right\|_{L^{1}} \\
\leqslant & \left\|v_{1 x x}(s)\right\|_{L^{2}}\left\|\left(v_{1 x}-v_{2 x}\right)(s)\right\|_{L^{2}} \sum_{i=0}^{p-1}\left\|v_{1 x}(s)\right\|_{L^{2}}^{i}\left\|v_{2 x}(s)\right\|_{L^{2}}^{p-1-i} \\
& +\left\|v_{2 x}(s)\right\|_{L^{2}}^{p}\left\|\left(v_{1 x x}-v_{2 x x}\right)(s)\right\|_{L^{2}} \\
\leqslant & C \delta^{p} M\left(v_{1}-v_{2}\right)(1+s)^{-2} \sum_{i=0}^{p-1}(1+s)^{-3(p-1) / 4}+C \delta^{p} M\left(v_{1}-v_{2}\right)(1+s)^{-2} \\
\leqslant & C \delta^{p} M\left(v_{1}-v_{2}\right)(p+1)(1+s)^{-2},
\end{aligned}
$$

where we used $-3(p-1) / 4<0$,

$$
\begin{aligned}
& \frac{1}{p+1} a_{p}\left\{p\left\|\theta^{p-1}(s) \theta_{x}(s)\left(v_{1 x}-v_{2 x}\right)(s)\right\|_{L^{1}}+\left\|\theta(s)^{p}\left(v_{1 x x}-v_{2 x x}\right)(s)\right\|_{L^{1}}\right\} \\
& \quad \leqslant p\|\theta(s)\|_{L^{\infty}}^{p-1}\left\|\theta_{x}(s)\right\|_{L^{2}}\left\|\left(v_{1 x}-v_{2 x}\right)(s)\right\|_{L^{2}} \\
& \quad+\|\theta(s)\|_{L^{\infty}}^{p-1}\|\theta(s)\|_{L^{2}}\left\|\left(v_{1 x x}-v_{2 x x}\right)(s)\right\|_{L^{2}} \\
& \quad \leqslant C\left\{p \eta^{p} M\left(v_{1}-v_{2}\right)(1+s)^{-(p+2) / 2}+\eta^{p} M\left(v_{1}-v_{2}\right)(1+s)^{-(p+2) / 2}\right\} \\
& \quad \leqslant C \eta^{p}(p+1) M\left(v_{1}-v_{2}\right)(1+s)^{-2},
\end{aligned}
$$

where $-(p+2) / 2 \leqslant-2$,

$$
\begin{aligned}
& \frac{1}{p+1} \sum_{j=1}^{p-1} a_{j} j\left\|\theta^{j-1}(s) \theta_{x}(s)\left(v_{1 x}^{p+1-j}-v_{2 x}^{p+1-j}\right)(s)\right\|_{L^{1}} \\
& \leqslant \frac{1}{p+1} \sum_{j=1}^{p-1} a_{j} j\|\theta(s)\|_{L^{\infty}}^{j-1}\left\|\theta_{x}(s)\right\|_{L^{\infty}}\left\|\left(v_{1 x}-v_{2 x}\right)(s)\right\|_{L^{2}} \\
& \quad \times \sum_{i=0}^{p-j}\left\|v_{1 x}(s)\right\|_{L^{2}}^{i}\left\|v_{2 x}(s)\right\|_{L^{2}}^{p-j-i}
\end{aligned}
$$




$$
\begin{aligned}
\leqslant & \frac{C}{p+1} \sum_{j=1}^{p-1} a_{j} j \eta^{j}(1+s)^{-(j-1) / 2-1} M\left(v_{1}-v_{2}\right)(1+s)^{-3 / 4} \\
& \times \sum_{i=0}^{p-j} \delta^{p-j}(1+s)^{-3(p-j) / 4} \\
\leqslant & C \frac{1}{p+1} \sum_{j=1}^{p-1} a_{j} j(p-j+1) \eta^{j} \delta^{p-j} M\left(v_{1}-v_{2}\right)(1+s)^{-(p+3) / 2} \\
\leqslant & C M\left(v_{1}-v_{2}\right)(1+s)^{-2} \frac{1}{p+1} \sum_{j=1}^{p-1} a_{j} j(p-j+1) \eta^{j} \delta^{p-j},
\end{aligned}
$$

where $-(3 p+5-j) / 4 \leqslant-(p+3) / 2$ for $1 \leqslant j \leqslant p-1$ and $-(p+3) / 2 \leqslant-2$ for $p \geqslant 2$, and

$$
\begin{aligned}
\frac{1}{p+1} & \sum_{j=1}^{p-1} a_{j}(p+1-j)\left\|\theta(s)^{j}\left(v_{1 x}^{p-j} v_{1 x x}-v_{2 x}^{p-j} v_{2 x x}\right)(s)\right\|_{L^{1}} \\
\leqslant & \frac{1}{p+1} \sum_{j=1}^{p-1} a_{j}(p+1-j)\|\theta(s)\|_{L^{\infty}}^{j}\left\{\left\|v_{1 x}(s)\right\|_{L^{2}}^{p-j}\left\|\left(v_{1 x x}-v_{2 x x}\right)(s)\right\|_{L^{2}}\right. \\
& \left.+\left\|v_{2 x x}(s)\right\|_{L^{2}}\left\|\left(v_{1 x}-v_{2 x}\right)(s)\right\|_{L^{2}} \sum_{i=0}^{p-j-1}\left\|v_{1 x}(s)\right\|_{L^{2}}^{i}\left\|v_{2 x}(s)\right\|_{L^{2}}^{p-j-1-i}\right\} \\
\leqslant & \frac{C}{p+1} \sum_{j=1}^{p-1} a_{j}(p+1-j) \eta^{j}(1+s)^{-j / 2}\left\{\delta^{p-j} M\left(v_{1}-v_{2}\right)(1+s)^{-(3 p+5-3 j) / 4}\right. \\
& \left.+\delta^{p-j} M\left(v_{1}-v_{2}\right)(1+s)^{-2}(1+s)^{-3(p-j-1) / 4}(p-j)\right\} \\
\leqslant & \frac{C}{p+1} \sum_{j=1}^{p-1} a_{j}(p+1-j) \eta^{j} \delta^{p-j} M\left(v_{1}-v_{2}\right)\left\{(1+s)^{-(3 p+5-j) / 4}\right. \\
& \left.+(1+s)^{-(3 p+5-j) / 4}(p-j)\right\} \\
\leqslant & C M\left(v_{1}-v_{2}\right)(1+s)^{-(p+3) / 2} \frac{1}{p+1} \sum_{j=1}^{p-1} a_{j}(p+1-j)^{2} \eta^{j} \delta^{p-j} \\
\leqslant & C M\left(v_{1}-v_{2}\right)(1+s)^{-2} \frac{1}{p+1} \sum_{j=1}^{p-1} a_{j}(p+1-j)^{2} \eta^{j} \delta^{p-j}
\end{aligned}
$$

where we used $-(3 p+5-j) / 4 \leqslant-(p+3) / 2 \leqslant-2$ for $1 \leqslant j \leqslant p-1$ and $p \geqslant$ 2 , it is easy to obtain 


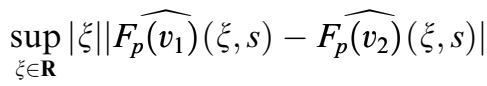

$$
\begin{aligned}
& \leqslant C M\left(v_{1}-v_{2}\right)(1+s)^{-2}\left\{\delta^{p}(p+1)+\eta^{p}(p+1)\right. \\
& \left.+\frac{1}{p+1} \sum_{j=1}^{p-1}\left(a_{j} j(p+1-j) \eta^{j} \delta^{p-j}+a_{j}(p+1-j)^{2} \eta^{j} \delta^{p-j}\right)\right\} \\
& \leqslant C(\eta+\delta)^{p} M\left(v_{1}-v_{2}\right)(1+s)^{-2} \text {. }
\end{aligned}
$$

With 3.38, 3.39, 3.40 and (3.46), we finish the proof of the lemma.

Proof of Theorem 3.1. Rewriting (3.7) as the operational form $v=S v$. We need to prove that there exists the positive constant $\delta_{1}$ such that the operator $S$ is a contraction mapping from $X_{p}^{\delta_{1}}$ into $X_{p}^{\delta_{1}}$.

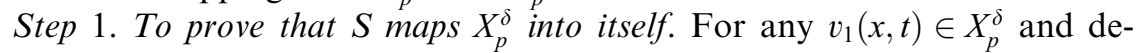
noting $v=S v_{1}$, we will prove $v \in X_{p}^{\delta}$ for some small $\delta>0$. By the definition of $M_{p}(v)$ and Lemma 3.5, for any positive integer $p$, there exist a constant $C_{1}$ such that

$$
M_{p}(v) \leqslant C_{1}\left(\left\|v_{0}\right\|_{W^{3,1}}+\eta+(\eta+\delta)^{p+1}\right) .
$$

Choose $\delta_{2} \leqslant 1 / n C_{1}$ with $n=\max \left\{2+2^{p+1}, 1 / C_{1}\right\}$. Then for $\left\|v_{0}\right\|_{W^{3,1}} \leqslant \delta_{2} / n C_{1}$, $\eta \leqslant \delta_{2} / n C_{1}$ and $\delta<\delta_{2}$, we have

$$
\begin{aligned}
M_{p}(v) & \leqslant C_{1}\left(\frac{\delta_{2}}{n C_{1}}+\frac{\delta_{2}}{n C_{1}}+\left(\frac{\delta_{2}}{n C_{1}}+\delta_{2}\right)^{p+1}\right) \\
& =C_{1}\left(\frac{2 \delta_{2}}{n C_{1}}+\delta_{2}^{p+1}\left(\frac{1}{n C_{1}}+1\right)^{p+1}\right) \\
& \leqslant C_{1}\left(\frac{2 \delta_{2}}{n C_{1}}+\delta_{2}^{2} 2^{p+1}\right) \\
& =C_{1} \frac{\left(2+2^{p+1}\right) \delta_{2}}{n C_{1}} \leqslant \delta_{2} .
\end{aligned}
$$

Thus, we proved $S: X_{p}^{\delta} \rightarrow X_{p}^{\delta}$ for some small $\delta<\delta_{2}$.

Step 2. To prove that $S$ is a contraction in $X_{p}^{\delta}$. Suppose that $v_{1}(x, t)$, $v_{2}(x, t) \in X_{p}^{\delta}\left(\delta<\delta_{2}\right)$. By (3.7),

$$
\begin{aligned}
\partial_{x}^{j}\left(S v_{1}-S v_{2}\right)= & \frac{1}{2 \pi} \int_{0}^{t} \int_{-\infty}^{\infty}(\mathrm{i} \xi)^{j} \mathrm{e}^{\mathrm{i} \xi x} \mathrm{e}^{-B(\xi)(t-s)} \\
& \times \frac{F_{p} \widehat{\left(v_{1}\right)}(\xi, s)-F_{p} \widehat{\left(v_{2}\right)}(\xi, s)}{1+\xi^{4}} \mathrm{~d} \xi \mathrm{d} s
\end{aligned}
$$

for $j=0,1,2$ and $p \geqslant 1$. First, we estimate $\left\|S v_{1}-S v_{2}\right\|_{L^{2}}$. 


$$
\begin{aligned}
\int_{0}^{t} & \left\|\frac{1}{2 \pi} \int_{-\infty}^{\infty} \mathrm{e}^{\mathrm{i} \xi} \frac{\mathrm{e}^{-B(\xi)(t-s)}}{1+\xi^{4}}\left(F_{p} \widehat{\left(v_{1}\right)}(\xi, s)-F_{p} \widehat{\left(v_{2}\right)}(\xi, s)\right) \mathrm{d} \xi\right\|_{L^{2}} \mathrm{~d} s \\
& =\int_{0}^{t}\left\|\frac{\mathrm{e}^{-B(\xi)(t-s)}}{\left.1+F_{p} \widehat{\left(v_{1}\right)}(\xi, s)-F_{p} \widehat{\left(v_{2}\right)}(\xi, s)\right)}\right\|_{L^{2}} \mathrm{~d} s \\
& =\int_{0}^{t}\left(\int_{-\infty}^{\infty} \frac{\mathrm{e}^{-2 B(\xi)(t-s)}}{\left(1+\xi^{4}\right)^{2}}\left|\widehat{F_{p}} \widehat{\left(v_{1}\right)}(\xi, s)-F_{p} \widehat{\left(v_{2}\right)}(\xi, s)\right|^{2} \mathrm{~d} \xi\right)^{1 / 2} \mathrm{~d} s \\
& \leqslant \int_{0}^{t} \sup _{\xi \in \mathbf{R}}\left|F_{p} \widehat{\left(v_{1}\right)}(\xi, s)-F_{p} \widehat{\left(v_{2}\right)}(\xi, s)\right|\left(\int_{-\infty}^{\infty} \frac{\mathrm{e}^{-2 B(\xi)(t-s)}}{\left(1+\xi^{4}\right)^{2}} \mathrm{~d} \xi\right)^{1 / 2} \mathrm{~d} s .
\end{aligned}
$$

Using Lemmas 3.2, 3.3 and 3.6, we obtain

$$
\begin{aligned}
& \int_{0}^{t}\left\|\frac{1}{2 \pi} \int_{-\infty}^{\infty} \mathrm{e}^{\mathrm{i} \xi x} \frac{\mathrm{e}^{-B(\xi)(t-s)}}{1+\xi^{4}}\left(\widehat{F_{1}\left(v_{1}\right)}(\xi, s)-\widehat{F_{1}} \widehat{\left(v_{2}\right)}(\xi, s)\right) \mathrm{d} \xi\right\|_{L^{2}} \mathrm{~d} s \\
& \quad \leqslant C(\eta+\delta) M\left(v_{1}-v_{2}\right) \int_{0}^{t}(1+s)^{-(1-\sigma)}(1+t-s)^{-1 / 4} \mathrm{~d} s \\
& \quad \leqslant C(\eta+\delta) M\left(v_{1}-v_{2}\right)(1+t)^{1-(1-\sigma)-1 / 4} \\
& \quad \leqslant C(\eta+\delta) M\left(v_{1}-v_{2}\right)(1+t)^{-(1 / 4-\sigma)}, \quad 0<\sigma \leqslant 1 / 2, \\
& \int_{0}^{t}\left\|\frac{1}{2 \pi} \int_{-\infty}^{\infty} \mathrm{e}^{\mathrm{i} \xi \xi x} \frac{\mathrm{e}^{-B(\xi)(t-s)}}{1+\xi^{4}}\left(F_{p} \widehat{\left(v_{1}\right)}(\xi, s)-\widehat{F_{p}} \widehat{\left(v_{2}\right)}(\xi, s)\right) \mathrm{d} \xi\right\|_{L^{2}} \mathrm{~d} s \\
& \quad \leqslant C(\eta+\delta)^{p} M\left(v_{1}-v_{2}\right) \int_{0}^{t}(1+s)^{-3 / 2}(1+t-s)^{-1 / 4} \mathrm{~d} s \\
& \quad \leqslant C(\eta+\delta)^{p} M\left(v_{1}-v_{2}\right)(1+t)^{-1 / 4}, \quad p \geqslant 2 .
\end{aligned}
$$

Namely, we obtain

$$
\left\|S v_{1}-S v_{2}\right\|_{L^{2}} \leqslant \begin{cases}C(\eta+\delta) M\left(v_{1}-v_{2}\right)(1+t)^{-(1 / 4-\sigma)}, & 0<\sigma<1 / 2 \\ C(\eta+\delta)^{p} M\left(v_{1}-v_{2}\right)(1+t)^{-1 / 4} & \text { for } p=1 \\ & \text { for } p \geqslant 2\end{cases}
$$

Using Lemmas 3.2, 3.3 and 3.6, we can similarly estimate $\left\|\partial_{x}\left(S v_{1}-S v_{2}\right)\right\|_{L^{2}}$ and $\left\|\partial_{x}^{2}\left(S v_{1}-S v_{2}\right)\right\|_{L^{2}}$ as follows:

$$
\left\|\partial_{x}\left(S v_{1}-S v_{2}\right)\right\|_{L^{2}} \leqslant \begin{cases}C(\eta+\delta) M\left(v_{1}-v_{2}\right)(1+t)^{-(3 / 4-\sigma)}, & 0<\sigma<1 / 2 \\ C(\eta+\delta)^{p} M\left(v_{1}-v_{2}\right)(1+t)^{-3 / 4} & \text { for } p=1, \\ \text { for } p \geqslant 2 .\end{cases}
$$


and

$$
\left\|\partial_{x}^{2}\left(S v_{1}-S v_{2}\right)\right\|_{L^{2}} \leqslant \begin{cases}C(\eta+\delta) M\left(v_{1}-v_{2}\right)(1+t)^{-(1-\sigma)}, & 0<\sigma<1 / 2 \\ C(\eta+\delta)^{p} M\left(v_{1}-v_{2}\right)(1+t)^{-5 / 4} & \text { for } p=1, \\ \text { for } p \geqslant 2 .\end{cases}
$$

With (3.50)-(3.52), we proved that, for some constant $C_{1}$,

$$
M\left(S v_{1}-S v_{2}\right) \leqslant C_{1}(\eta+\delta)^{p} M\left(v_{1}-v_{2}\right) .
$$

Choose $\delta \leqslant \delta_{3}<1 / n C_{1}$ with $n=\max \left\{2 / C_{1}, 2\right\}$, then for $\eta<\delta_{3}, M\left(v_{1}\right)<\delta_{3}$ and $M\left(v_{2}\right)<\delta_{3}$, we have

$$
M\left(S v_{1}-S v_{2}\right)<M\left(v_{1}-v_{2}\right) .
$$

That is $S: X_{p}^{\delta} \rightarrow X_{p}^{\delta}$ is contracting for some small $\delta<\delta_{3}$.

By Steps 1 and 2 , let $\delta_{1}<\min \left\{\delta_{2}, \delta_{3}\right\}$, we have proved that the operator $S$ is contracting from $X_{p}^{\delta_{1}}$ to $X_{p}^{\delta_{1}}$. By Banach's fixed point theorem, $S$ has a unique fixed point $v(x, t)$ in $X_{p}^{\delta_{1}}$. Thus, the integral equation has a unique global solution $v(x, t) \in X_{p}^{\delta_{1}}$.

\section{Numerical simulations}

In order to confirm the theoretical result in Theorem 2.2, numerical simulation has been carried out for cases $p=1$ and $p=3$. The applied numerical scheme solving Eqs. (1.1) and (2.3) is the explicit finite difference scheme. For the consideration of the Cauchy problem, we adopt a suitable IBVP for a sufficiently large domain $\Omega \subset \mathbf{R}$ with zero Neumann boundary in stead of the original IVP.

Let $\theta(x, t)$ and $u(x, t)$ denote the solutions of the parabolic equation and the R-B equation, respectively. Consider the initial condition $\theta_{0}(x)$ and $u_{0}(t)$ as

$$
\theta_{0}(x)=u_{0}(x)=\mathrm{e}^{-x^{2}} .
$$

Thus,

$$
\int_{-\infty}^{\infty} \theta_{0}(x) \mathrm{d} x=\int_{-\infty}^{\infty} u_{0}(x) \mathrm{d} x=\int_{-\infty}^{\infty} \mathrm{e}^{-x^{2}} \mathrm{~d} x>0 .
$$

But $\int_{-\infty}^{\infty}\left[\theta_{0}(x)-u_{0}(x)\right] \mathrm{d} x=0$

For the following discussion, the time-step and the space-step are 0.5 and 1 , respectively. We compute the time $t$ from 0 to 20000 for $x \in \Omega=[-2000,2000]$.

\subsection{Case $p=1$}

The solution of the $\mathrm{R}-\mathrm{B}$ equation for $t \in[2000,10000]$ and $x \in[-200,200]$ is displayed in Fig. 1. The grid size used in plotting the graph is $\Delta x=4$ and $\Delta t=100$. The diffusion wave solution of the corresponding nonlinear parabolic equation is similar to Fig. 1. Fig. 2 shows the difference of these two 




Fig. 1. The solution of the $\mathrm{R}-\mathrm{B}$ equation.

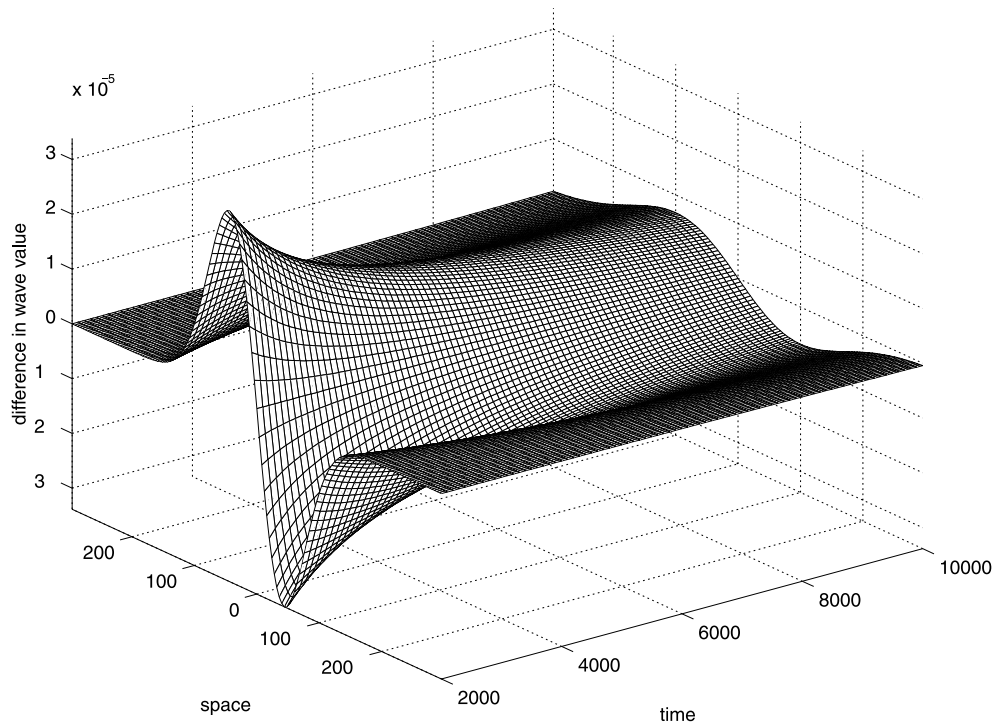

Fig. 2. The difference of the solutions $u(x, t)-\theta(x, t)$.

solutions: $u(x, t)-\theta(x, t)$ with the same time and space intervals, and the same grid as in Fig. 1. Comparing Figs. 1 and 2, noticing the different scales in the $Z$-axes, we discover that the value of $u(x, t)-\theta(x, t)$ is much smaller than 

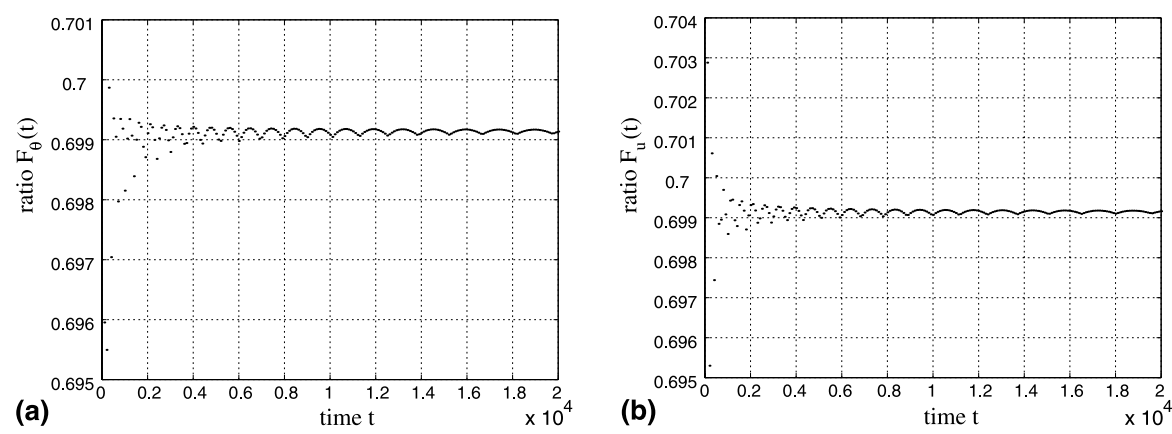

Fig. 3. (a) The ratio $F_{\theta}(t)=\|\theta(x, t)\|_{L^{\infty}} /(1+t)^{-1 / 2}$. (b) The ratio $F_{u}(t)=\|u(x, t)\|_{L^{\infty}} /(1+t)^{-1 / 2}$.

$u(x, t)-0$. Thus, we expect that $u(x, t)$ approaches to $\theta(x, t)$ much faster than it does to zero. Since $u(x, t), \theta(x, t)$ and $u(x, t)-\theta(x, t)$ approach to zero when $x$ approaches to infinity, $\|u(t)\|_{L^{\infty}},\|\theta(t)\|_{L^{\infty}}$ and $\|(u-\theta)(t)\|_{L^{\infty}}$ can be estimated by $\max _{x \in \Omega}|u(x, t)|, \max _{x \in \Omega}|\theta(x, t)|$ and $\max _{x \in \Omega}|u(x, t)-\theta(x, t)|$, respectively. Figs. 3, 4, 6 present the decay rates for $u(x, t), \theta(x, t)$ and $u(x, t)-\theta(x, t)$. The grid size used in these figures is $\Delta t=100$.

Fig. 3(a) shows the ratio $F_{\theta}(t)=\|\theta(t)\|_{L^{\infty}} /(1+t)^{-1 / 2}$. For the time $t$ from 10,000 to 20,000, we obtain almost a straight line. This implies $F_{\theta}(t) \rightarrow C$ when $t \rightarrow \infty$, where $C=0.6992$. Thus, we have $\|\theta(t)\|_{L^{\infty}}=C(1+t)^{-1 / 2}$, which confirms the theoretical assertion: $\|\theta(t)\|_{L^{\infty}} \leqslant C(1+t)^{-1 / 2}$. Similar result can be found for $\|u(t)\|_{L^{\infty}}$ from the ratio $F_{u}(t)=\|\theta(t)\|_{L^{\infty}} /(1+t)^{-1 / 2}$ shown in Fig. 3(b).

Our main interest is the decay rate of the difference $u(x, t)-\theta(x, t)$. The ratio $F_{s}(t)=\|(u-\theta)(t)\|_{L^{\infty}} /(1+t)^{-7 / 8}$ is shown in Fig. 4(a), where the ratio value keeps decreasing as $t$ increases. This numerical result shows that $\|(u-\theta)(t)\|_{L^{\infty}}$

(a)



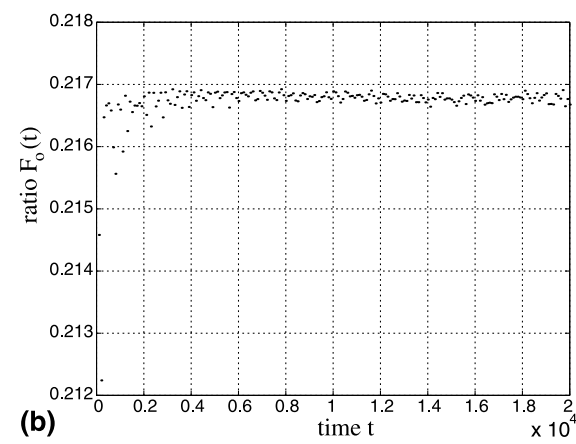

(b)

Fig. 4. (a) The ratio $F_{s}(t)=\|u(x, t)-\theta(x, t)\|_{L^{\infty}} /(1+t)^{-7 / 8}$ and (b) the ratio $F_{s}(t)=\| u(x, t)-$ $\theta(x, t) \|_{L^{\infty}} /(1+t)^{-0.998}$. 

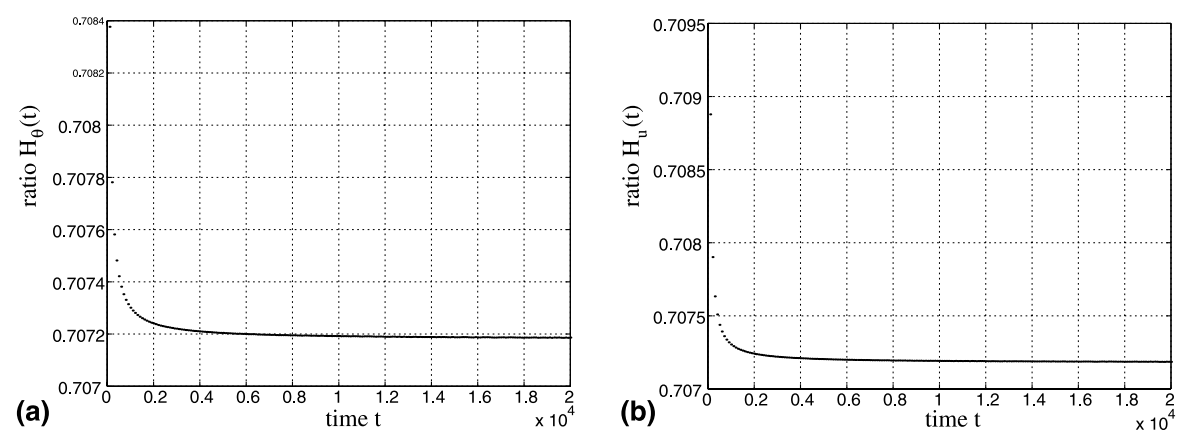

Fig. 5. (a) The ratio $H_{\theta}(t)=\|\theta(x, t)\|_{L^{\infty}} /(1+t)^{-1 / 2}$. (b) The ratio $H_{u}(t)=\|u(x, t)\|_{L^{\infty}} /(1+t)^{-1 / 2}$.

decays faster than the function $(1+t)^{-7 / 8}$. In fact, it is found that $\|(u-$ $\theta)(t) \|_{L^{\infty}}$ decays to zero with the same rate as $(1+t)^{-0.998}$, which is shown in Fig. 4(b). Thus, in this example, $\|(u-\theta)(t)\|_{L^{\infty}}=C(1+t)^{-0.998}$ with $C=$ 0.2168. Several other initial conditions have been considered. However, we have not found any example such that $\|(u-\theta)(t)\|_{L^{\infty}}=C(1+t)^{-7 / 8+\sigma}$ (with $\sigma>0)$. This may imply that our theoretical result is not optimal. Inspired by Fig. 4(b), it seems that $\|(u-\theta)(t)\|_{L^{\infty}}=C(1+t)^{-1}$, whose theoretical proof still remains open.

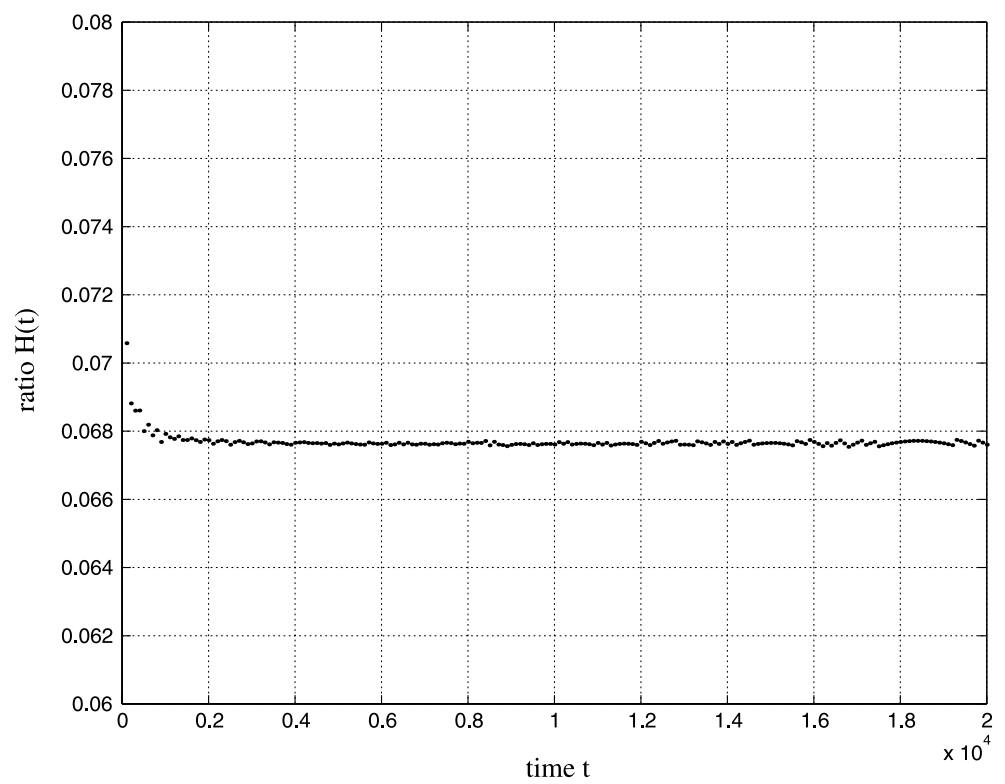

Fig. 6. The ratio $H(t)=\|u(x, t)-\theta(x, t)\|_{L^{\infty}} /(1+t)^{-1}$. 


\subsection{Case $p=3$}

The theoretical result for cases $p \geqslant 2$ are the same, numerically we only present the results for $p=3$. For the considered initial condition, the decay rates of $u(x, t)$ and $\theta(x, t)$ are displayed in Fig. 5(a) and (b), respectively. In Fig. 6 , as $t \rightarrow \infty$, the ratio line of $\|(u-\theta)(t)\|_{L^{\infty}} /(1+t)^{-1}$ seems to be a constant line, implying $\|(u-\theta)(t)\|_{L^{\infty}} /(1+t)^{-1} \rightarrow C$, where $C=0.0675$. Hence, $\|(u-\theta)(t)\|_{L^{\infty}}$ decays as fast as the function $(1+t)^{-1}$ does, confirming our theoretical result.

\section{Acknowledgements}

The first author would like to acknowledge the strong support and valuable suggestions received from her supervisor Professor Yau-Shu Wong.

\section{References}

[1] M. Mei, Long-time behavior of solution for Rosenau-Burgers equation (I), Appl. Anal. 63 (1996) 315-330.

[2] M. Mei, Long-time behavior of solution for Rosenau-Burgers equation (II), Appl. Anal. 68 (1998) 333-356.

[3] P. Rosenau, Dynamics of dense discrete systems, Prog. Theor. Phys. 79 (1988) 1028-1042.

[4] M.A. Park Model equations in fluid dynamics, Ph.D. Dissertation, Tulane University, 1990.

[5] S.K. Chung, S.H. Ha, Finite element Garlerkin solutions for the Rosenau equation, Appl. Anal. 54 (1994) 39-56.

[6] S. Kinami, M. Mei, S. Omata, Convergence to diffusion waves of the solutions for BenjaminBona-Mahony-Burgers equations, Appl. Anal. 75 (2000) 317-340.

[7] M. Mei, C. Schmeiser, Aspmptotic profiles of solutions for the BBM-Burgers equation, Funkcial. Ekvac. 44 (2001) 151-170.

[8] I.L. Chern, T.P. Liu, Convergence to diffusion waves of solutions for viscous conservation laws, Comm. Math. Phys. 110 (1987) 503-517.

[9] I.L. Chern, T.P. Liu, Convergence to diffusion waves of solutions for viscous conservation laws, Comm. Math. Phys. 120 (1989) 525-527.

[10] A. Jeffrey, H. Zhao, Global existence and optimal temporal decay estimates for system parabolic conservation laws. I. The one-dimensional case, Appl. Anal. 70 (1998) 175-193.

[11] A. Matsumura, On the asymptotic behavior of solutions of semi-linear wave equation, Publ. RIMS Kyoto Univ. 12 (1976) 169-189.

[12] I.E. Segal, Quantization and disperson for nonlinear relativistic equations, Mathematical Theory of Elementary Particles, MIT Press, Cambridge, MA, 1966, pp. 79-108. 